\title{
Multimodal imaging in retinoschisis $\mathrm{X}$-linked.
}

\section{Gilda Cennamo MD ${ }^{1 *}$, Nicola Centore MD², Federica Mirra MD², Laura Pavese MD $^{2}$, Giuseppe de Crecchio MD $^{2}$}

${ }^{1}$ Department of Public Health, Federico II university of Naples, Italy

${ }^{2}$ Department of Neurosciences, University of Naples Federico II, Italy

\begin{abstract}
To report retinal alterations of a X-Linked Juvenile Retinoschisis (XLRS) case of with multimodal imaging. This case report highlights the role of multimodal imaging examining vascular and structural alterations under this condition through non-invasive imaging techniques to help with making the diagnosis and follow up.
\end{abstract}

Keywords: Diagnosis, multimodal imaging, vitro retinal disorder, optical coherence tomography

Accepted on March 13, 2018

\section{Introduction}

Congenital Retinoschisis (XLRS) is a rare bilateral vitreoretinal disorder transmitted as an $\mathrm{X}$ linked recessive trait associated with a mutation of the XLRS1 gene located on the short arm of the $\mathrm{X}$ chromosome, $\mathrm{Xp} 22$ [1]. The estimated prevalence is up to 1 in 20,000 with a bilateral appearance in $40 \%$ of the patients [2]. Clinically, the patients show typically a cystic-like stellate maculopathy or a foveal schisis and peripheral retinoschisis of various degrees of severity on fundoscopy [3]. Other authors described this condition in Optical Coherence Tomography (OCT) and OCT angiography (OCTA) as splitting of inner retinal layers and perifoveal microvascular changes, more evident in the deep vascular plexus [4]. The splitting of the inner retinal layers, mainly involving Inner Nuclear Layer (INL) followed by Outer Plexiform Layer (OPL) and ganglion cell layer (GCL), determines the formation of foveal schisis that leads to early bilateral visual loss [5]. In addition, XLRS may be complicated by retinal detachment, vitreous or intraretinal hemorrhage and vascualarized vitreous veils [6,7]. The purpose of our study is to describe XLRS patient with multimodal imaging modality including Spectral-Domain OCT (SD-OCT), En-face OCT, OCTA and fundus autofluorescence.

\section{Case Presentation}

We will take into consideration and describe the case of a 19 year-old male with juvenile retinoschisis whose Best Correct Visual Acuity was 20/50 in the right eye and 20/40 in the left one. Multicolor images (Heidelberg Engineering, Heidelberg, Germany) detect cartwheel-like macular degeneration (Figure 1A). Auto fluorescence (Heidelberg Engineering, Heidelberg, Germany) images reveal cystoid hyper auto fluorescence spaces (Figure 1B). Spectral Domain OCT (heidelberg spectral domain Optical Coherence Tomography (OCT) shows cystoid spaces in Ganglion Cell Layer (GCL), Inner Nuclear Layer ( INL), Outer

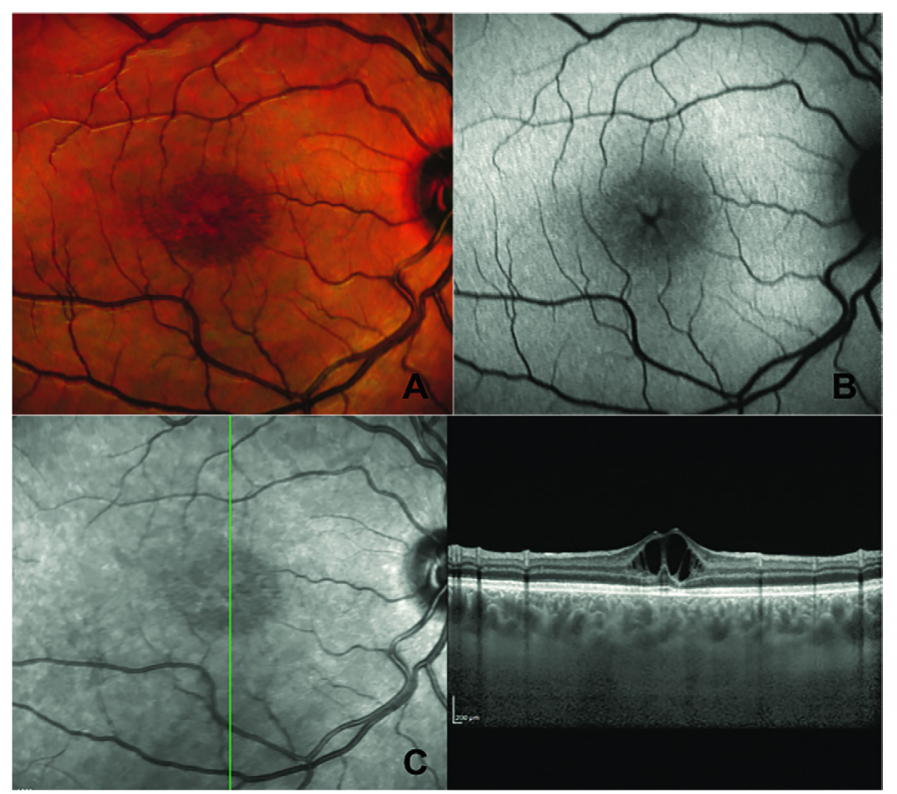

Figure 1: a) Multicolor images of the right eye demonstrate cartwheel-like macular Degeneration. b) Autofluorescence images reveal cystoid iperautofluorescence spaces c) Spectral Domain Optical Coherence Tomography shows cystoid spaces in the inner layers 

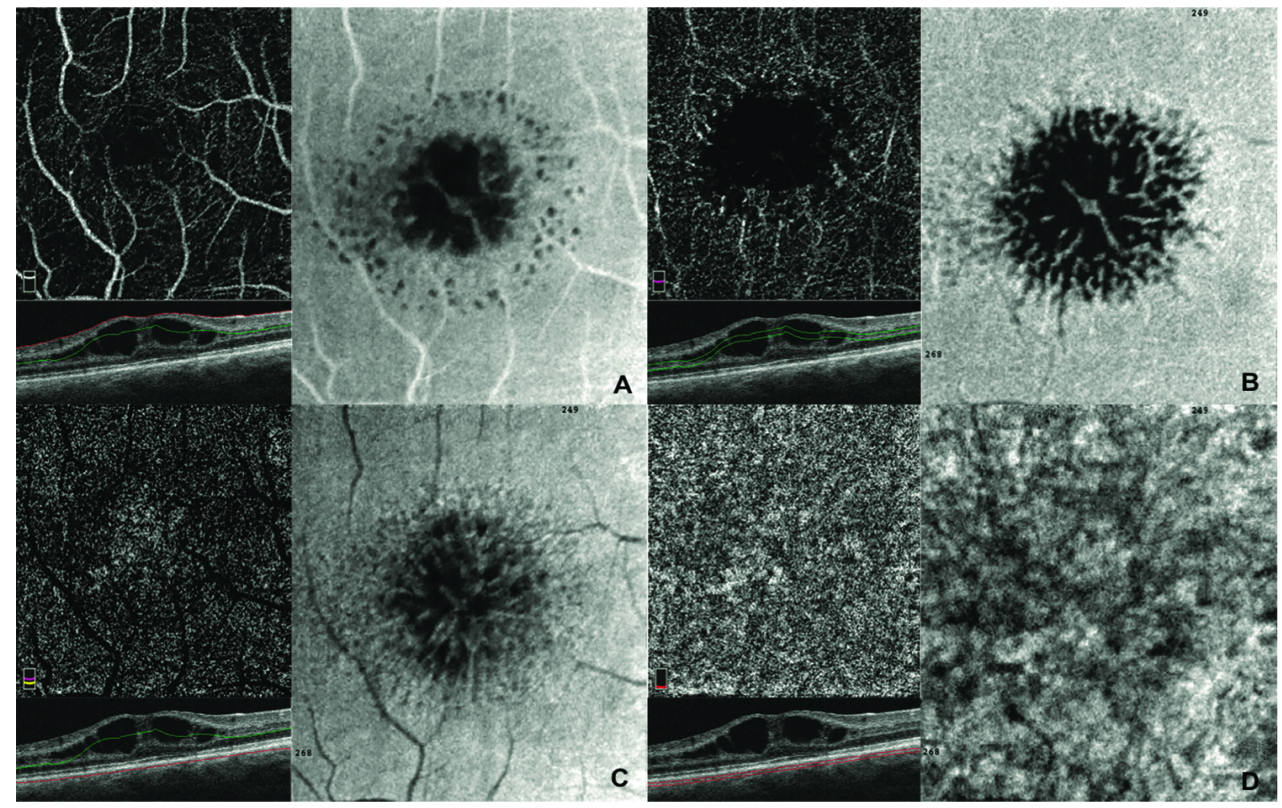

Figure 2: $(a, b, c, d)$ OCT angiography and En face OCT of right eye show an increase of foveal

A vascular zone in both superficial and deep vascular plexus, coral shape image in the inner retina and hypo hyper-reflectivity of the choroid layer.

Plexiform Layer (OPL) and the Outer Nuclear Layer (ONL). In addition, central macular and choroidal thickness have increased (Figure 1C). In agreement with other authors, OCTA (Angiovue System, Optovue Inc. Fremont, CA, USA) shows an increase of the foveal avascular zone in both superficial and deep vascular plexus and the presence of teleangectasias (Figure 2A and 2B) [4-8]. In Face images reveal the presence of reflective areas that appear like a petaoloid or coral shape without coriocapillaris alteration (Figure 2A-2C). Choriocapillaris images demonstrate hypo hyper-reflective areas due to a shadow effect without the presence of any vascular alteration (Figure 2D).

\section{Discussion}

Multimodal imaging enables to provide different but complementary information on $\mathrm{X}$ linked juvenile retinoschisis affecting both the inner and outer retinal structures. In our study, the SD-OCT analysis shows that schisis involves the GCL, INL, OPL and ONL. Other reports have highlighted that the most prevalent area of schisis is INL followed by GCL and OPL [5-9] Nan-Kai Wang et al. have noted the typical auto fluorescence pattern of hyper- and hypo-auto fluorescence in the macular area [10] Our findings show the appearance of macular schisis visible to auto fluorescence like a cystoid hyper auto fluorescence spaces, expression of changed light transmission. In agreement with previous studies, we have observed on OCTA images an increase of foveal avascular zone in both superficial and deep neurovascular plexus and we have highlighted vascular abnormalities such as teleangectasias like micro-vascular changes and capillary dropout rarefaction. These alterations of perifoveal microvasculature are described especially in the perifoveal deep vascular plexus due to the presence of schisis, accounting for possible biomarkers of disease prognosis. Moreover, Stanga et al. described a foveal petaloid a reflective area [8] and Tomoyo Yoshida-Uemura et al. described characteristic schisis patterns hyporeflective holes and a spoke-like pattern respectively in parafoveal and foveal region in GCL, a reticular pattern in INL and hyporeflective polygonal cavities in OPL [9-11]. In our case, foveal schisis appears on enface OCT like a petaoloid or coral shape without coriocapillaris alteration. In the present case, multimodal imaging will shed light on the in vivo structure of this rare condition offering more tools for its diagnosis, identifying typical features and helping in the follow up. Further experience with OCTA may clarify the precise role of this technology in diagnosing and managing retinal diseases.

\section{Acknowledgement}

The authors have no commercial or proprietary interest in any of the products or methods described in this article and they have not received any financial support for the performance of this study.

\section{References}

1. Sauer CG, Gehrig A. Positional cloning of the gene associated with X-linked juvenile retinoschisis. Nat Genet. 1997;17:164-70.

2. Kim DY, Mukai S. X-linked juvenile retinoschisis (XLRS): a review of genotype-phenotype relationships. Semin Ophthalmol. 2013;28:392-6.

3. Simonelli F, Cennamo G, Ziviello C, et al. Clinical features of $\mathrm{X}$ linked juvenile retinoschisis associated with new mutations in the XLRS1 gene in Italian families. Br J Ophthalmol. 2003;87:1130-4.

4. Stringa F, Tsamis E, Papayannis A, et al. Segmented swept source optical coherence tomography angiography assessment of the perifoveal vasculature in patients with X-linked juvenile retinoschisis a serial case report. 2017;10:329-35.

5. Padrón-Pérez N, Catalària J, Díaz J, et al. Swept-source and optical coherence tomography angiography in patients with X-linked retinoschisis. Eye. 2018;1:1-5. 
6. Kim DY, Mukai S. X-linked juvenile ret inoschisis. A review of genotype- phenotype relationships. Semin Ophthalmol. 2013;28:392-6.

7. Gregori NZ, Berrocal AM, Gregori G, et al. Macular spectraldomain optical coherence tomography in patients with $\mathrm{X}$ linked retinoschisis. Br J Ophthalmol. 2009;93:373-8.

8. Stanga EP, Papayannisa A, Tsamisa E, et al. Swept-Source Optical Coherence Tomography Angiography of Paediatric Macular Diseases. OCT Angiography in Retinal and Macular Diseases. Dev Ophthalmol. 2016;56:166-173.
9. Hu QR, Huang LZ, Chen XL, et al. X linked retinoshisis $\mathrm{X}$-Linked Retinoschisis in Juveniles Follow-Up by Optical Coherence Tomography Biomed Res Int. 2017;1:6-23.

10. Wang MK, Liu L, Chen HM, et al. Clinical presentations of X-linked retinoschisis in Taiwanese patients confirmed with genetic sequencing Molvisn. 2015;21:487-501.

11. Tomoyo Uemura T, Katagiri S, Yokoi T, et al. Different foveal schisis patterns in each retinal layer in eyes with hereditary juvenile retinoschisis evaluated by en-face optical coherence tomography. Graefes Arch Clin Exp Ophthalmol. 2015;255;4:719-23.

\section{*Correspondence to:}

Gilda Cennamo,

Department of Public Health, Federico II university of Naples, Italy

Mobile: +00390817463731

Fax: 00390817462383

E-mail: xgilda@hotmail.com 